\title{
Lessons from Explaining "Compatibility" to college students for Instrumental Education with FTIR as an Example
}

\author{
Yao-Yao Yang*, Chen-Wei Gu, Jian Chen, Geng-Yuan Zhang, Dengguang YU \\ School of Materials Science and Engineering, University of Shanghai for Science and Technology, \\ 516 Jungong Road, Shanghai 200093, China. \\ Email: yyyang@usst.edu.cn \\ *Corresponding author
}

\begin{abstract}
Instrumental education is a very important part in colleges and universities, which has a profound influence on cultivating the ability of undergraduates, particularly the ability of combine theory with practice. In this paper, compatibility, as a key hidden element in the design of suitable materials, particularly composite materials, is explored as a professional term in instrumental education. The clear disclosure and explanations of "compatibility" to college students should be beneficial to improve students' comprehensive ability, particularly the ability of "combining theory with practice". Although only Fourier-transform infrared spectroscopy (FTIR) has been innovatively developed here, a series of other characterization methods can be similarly explored as teaching materials for cultivating students' comprehensive ability during the scientific instrumental educations processes.
\end{abstract}

Keywords-Instrumental education; Higher School; Engineering education; Compatibility; Practical ability

\section{BACKGROUND}

The issue of students having influence and responsibility over their scientific instrumental learning is a complex issue [1]. Both lessons for explaining difficult theories and those for real experimental practices can be integrated together into the scientific instrumental educations. Thus, instrumental educations should be taken full advantages for cultivating the college students' ability.

A scientific instrument is, broadly speaking, a device or tool used for scientific purposes, including the study of both natural phenomena and theoretical research. In the universities for science and technology, there are often a wide variety of different kinds of scientific instruments. Learning to use these instruments is important for the students to become a valuable labor, become an excellent engineer or a scientific researcher in future in the society. On the other hand, the teachers should pay more attention to explore these instruments as tools for effective instrumental educations.

Compatibility is a measure of how stable a substance is when mixed with another substance [2-5]. If two substances can mix together and undergo a chemical reaction, they are considered incompatible. Today, man-made nanocomposites, which often consist of two, three or even more components are very popular both in laboratory and industrial applications. Correspondingly, the compatibility among the components frequently comprise a big concern in the related material development [6-7].

In analysis chemistry, many methods can be exploited to characterize the compatibility and the related stability of a nanocomposite [8-10]. Among these methods, Fouriertransform infrared spectroscopy (FTIR) is a technique used to obtain an infrared spectrum of absorption or emission of a solid, liquid or gas. An FTIR spectrometer simultaneously collects high-spectral-resolution data over a wide spectral range, often from 500 to $4000 \mathrm{~cm}^{-1}$. The term Fouriertransform infrared spectroscopy originates from the fact that a Fourier transform (a mathematical process) is required to convert the raw data into the actual spectrum. Infrared spectrometers are used to analyse molecular structure of samples qualitatively and /or quantitatively, without destruction of the molecules [11-15].

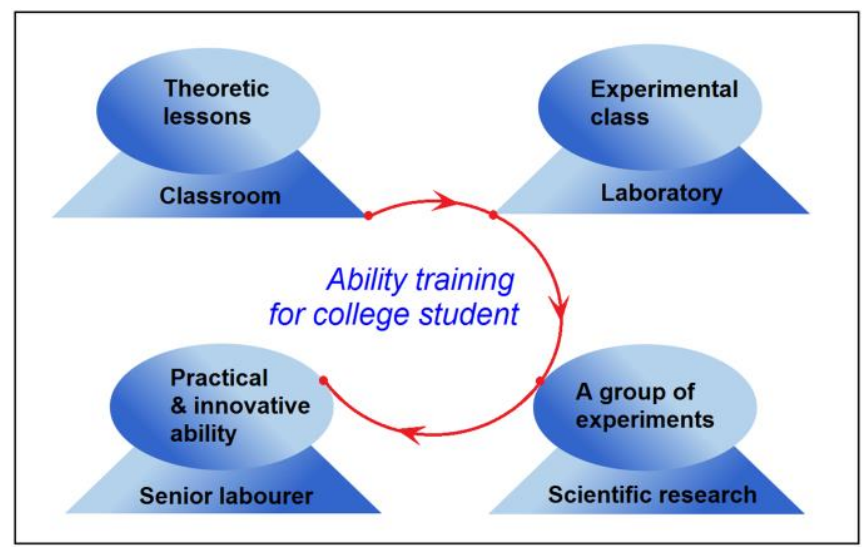

Fig. 1 Based on the instrumental education in higher school, a new procedure can be developed for improving the comprehensive ability of college students: i.e. from theoretical lessons in classroom, to a series of experimental class about instruments in laboratory, to a group of experiments aimed to one joint goal, and finally to cultivate their practical and innovative ability for sending a senior labourer to the society. 
Instrumental education occurs in lessons about analysis chemistry and also many other discipline. It can be excellent teaching materials for fostering the comprehensive ability of the college students in higher school (Fig. 1). The procedure include several steps, including from theoretical lessons in classroom, to a series of experimental class about instruments in laboratory, to a group of experiments aimed to one joint goal, and finally to cultivate their practical and innovative ability for sending a senior labourer to the society. Apparently, these steps reflect an advance gradually in due order, moving forward from simple to complex, from classroom to laboratory and to real life, and from theory to practice.

Each FT (Fourrier Transform) spectrometer is basically composed of the following optical elements: an IR source, an interferometer (with part of it, a beamsplitter) and a detector. In the FT machine, the beam produced by the source passes through an aperture, then eventually through an optical filter, and penetrates the inetrerometer. When the beam exits the interferometer, it is modulated by the scanner (moving mirror) The beam is then led to the sample (where it transmitted or reflected), and finally it is focused on the detector. In FTIR analyses, the incident beam is infrared light, which is composed of radiations. When a radiation beam strikes any object, it can be absorbed, transmitted, reflected, scattered, or it can excite fluorescence (Fig. 2). The achieved FTIR spectra can tell the information about the favorable secondary interactions between the coexistent components, and thus their compatibility for long-time period stability and functional performances.

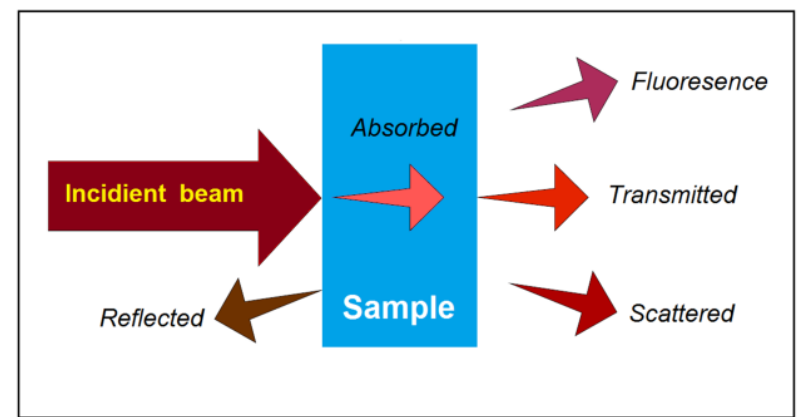

Fig. 2 FTIR spectra can tell the information about the favorable secondary interactions between the coexistent components, and thus their compatibility for long-time period stability and functional performances.

\section{II. “COMPATIBILITY” IS THE KEY HIDDEN ELEMENT FOR A SUITABLE MATERIAL DESIGN, PARTICULARLY THE COMPOSITE MATERIALS}

"Compatibility" is the key hidden element for a suitable material design, particularly the composite materials. Often, the suitable design needs a full and strong support of the practical experiences. A most famous example is the composition theory of a prescription in traditional Chinese medicine, i.e. "Monarch, Minister, Assistant and Guide". In the field of materials science and engineering, numerous double-component composites and multiple-component composites have this concern [16-19]. Particularly in today nano era, nanocomposites from a wide variety of generation methods need to be characterized about their chemical stability, physical stability and also physiological stability. From this standpoint, "compatibility" can be excellent supporting point for organizing engineering and innovation teaching material in a broad sense.

With medicated nanoparticles prepared using an electrospraying process as models, the role and place of compatibility is shown in Fig. 3. For the design of a new kind of medicated material, a model drug and its polymer should be reasonably selected, and sometimes the surfactant and transmembrane enhancer should be also selected for enhancing the functional performances of the final medicated material. Medicated materials at nano scale can provide many advantages over the traditional materials. Thus, a series of processes have been reported for developing nano medicated nanocomposites. Among them, electrospraying and electrospinning are the most popular "top-down" fabrication methods, which can transfer the working fluids into solid nanoparticles or nanofiber mats, respectively.

The characterizations of developed new products often consist of a series of analysis methods, which are exploited to disclose all their properties and performances, such as morphology, inner structure, physical forms and compatibility, mechanical/thermal/conductive/hydrophilic properties, and the desired functional performances. Through these experimental data, the design and selection of raw materials can be evaluated correctly. Certainly, among these evaluated parameters, "compatibility" is the key hidden element for the medicated composite materials. This is because that compatibility between the drug and its carrier is very important for the composite particles' functional performances and particularly, their stability.

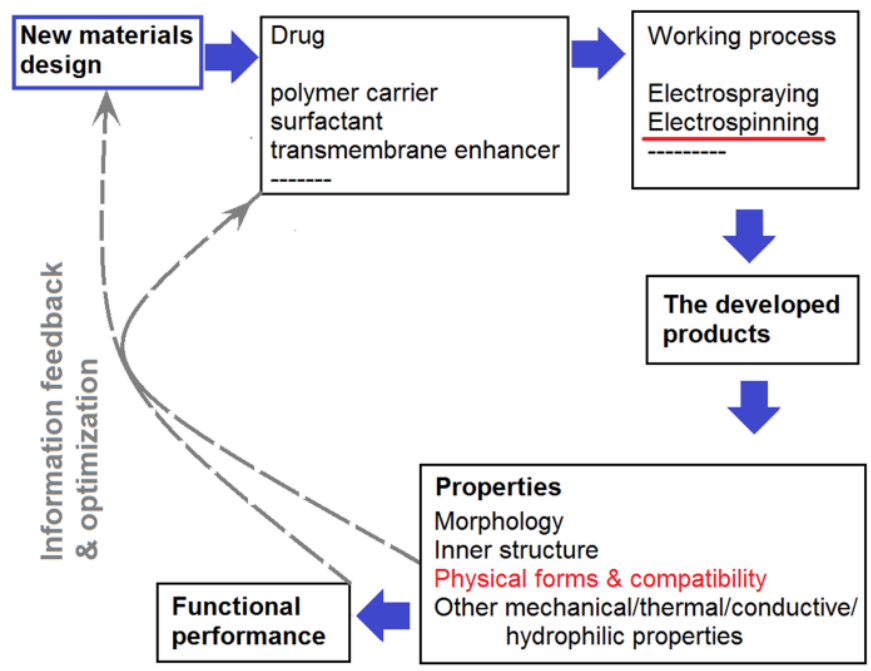

Fig. 3 Compatibility \& physical forms are important properties of the final medicated nanoproducts. 


\section{HIGH CORRELATION BETWEEN THE “COMPATIBILITY” AND THE INSTRUMENTAL ANALYSES}

"Compatibility" is an abstract concept, which is not easy to be explained as clearly as the exploited instruments. But this concept has a very close relationship with a series of instrumental analyses [20-21]. Good compatibility is prerequisite for the designed materials, for their storage or reactions, so that the vessel and other apparatus will not be damaged by its contents.

Often, secondary interactions between the components in the amorphous composites are very important for their stable coexistence, preventing the re-crystallization of drug molecules and also the possible solid phase separation within the solid particles [22-25]. For disclosing the potential secondary interactions between the components, FTIR is one of the most important and broadest methods for detecting the potential secondary interactions, such as the hydrogen bonding and hydrophobic interactions, which can be speculated from the appearance or disappearance of absorbance peaks, blue or red shifts of some characteristic peaks of the raw materials. Shown in Fig. 4, FTIR spectra can tell the information about the secondary interactions between the coexistent components such as hydrogen bonding, hydrophobic interactions, electrostatic interactions and also Van der Waals' force [2627]. These interactions are favorable for the stability of the final products, which can be evaluated using accelerated ageing tests.

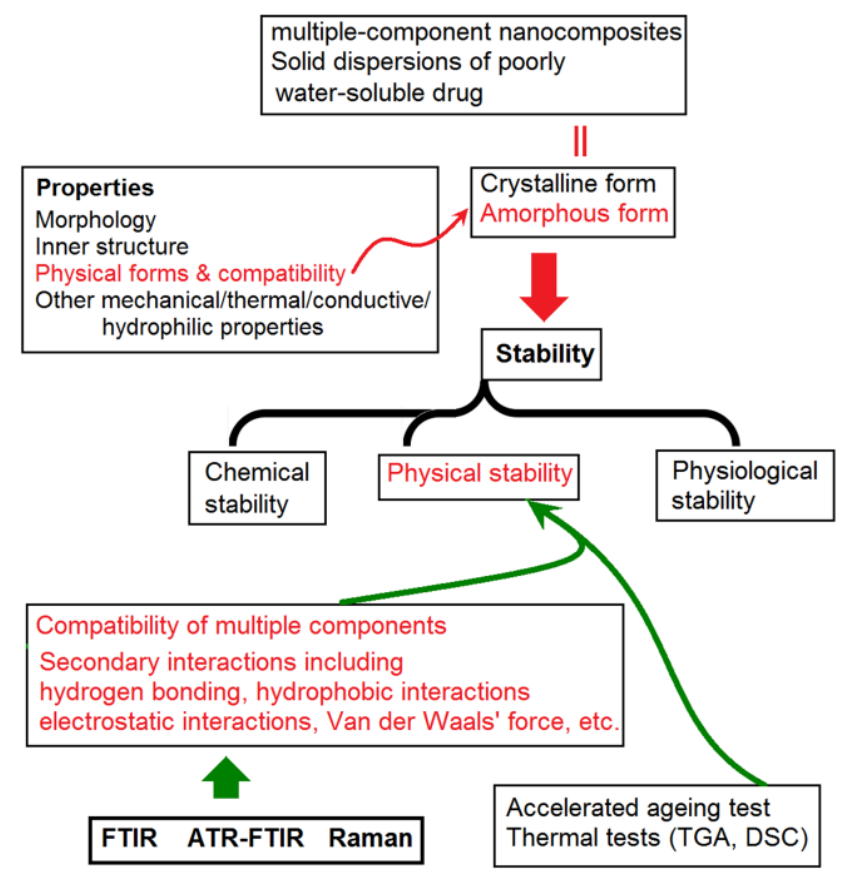

Fig. 4 FTIR spectra can tell the information about the favorable secondary interactions between the coexistent components, and thus their compatibility for long-time period stability and functional performances.

\section{CLEAR DiSClOSURE AND EXPLANATIONS OF “COMPATIBILITY” IN INSTRUMENTAL EDUCATION IS BENEFICIAL FOR THE STUDENTS TO IMPROVE THEIR COMPREHENSIVE ABILITY}

In all the universities of science and technology, instrumental education is very important key element for engineering education. It should create a profound influence on cultivating the undergraduate students' ability, particularly the capability of linking theory with practice. Clear disclose and explanations of "compatibility" in instrumental education is a very useful platform for improving the students' comprehensive ability. Particularly, "linking theory with practice" can be clearly exhibited to the college students majoring in materials science and engineering through theoretical explanations in classroom, to instrumental education with FTIR machines for understanding the working mechanism of this analysis method, and to a series of instrumental education on characterizing a new advanced material. Similarly with the FTIR, a series of other instrumental educations about scanning electron microscope (SEM), transmission electron microscope (TEM), X-ray diffraction (XRD), Raman spectroscopy (RM), and differential scanning calorimeter (DSC) can be explored to cultivate the students' ability of practice and innovation (Fig. 5) [28,29].

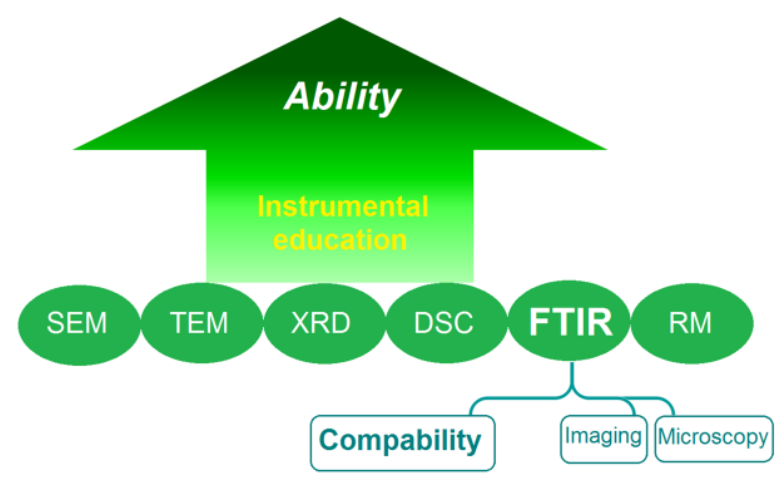

Fig. 5 Besides FTIR spectra, a series of other instrumental educations can be explored to cultivate the students' ability of practice and innovation.

\section{SUMMARY}

Scientific instrumental education can be a bridge to connect the lessons for explaining difficult theories and those for real experimental practices. The full usage of instrumental educations should be very useful for fostering the students' comprehensive ability, particularly the ability of "linking theory with practice". With compatibility as a professional term and Fourier-transform infrared spectroscopy (FTIR) as a teaching instrument, respectively, this paper successfully explains why and how the clear disclose and explanations of "compatibility" to the college students can be beneficial for them to improve their innovation and engineering ability. The developed teaching procedure can be similarly exploited in the lessons about other scientific instruments. 


\section{ACKNOWLEDGMENT}

The financial supports from the following projects are appreciated: the Shanghai Education Science Research Project (C17058), the National Natural Science Foundation of China (No. 51373101), the 2017 Graduate curriculum reform project in USST, and the College Student Innovation Project of USST (Nos.SH2018).

\section{REFERENCES}

[1] H. Jorgensen, "Student learning in higher instrumental education: who is responsible?" British J. Music Edu., vol.17, pp.67-77, 2000.

[2] Z. Zhang, W. Li, G. Wang, Y.L. Qu, and D.G. Yu, "Electrospun 4th generation solid dispersions of poorly water-soluble drug utilizing two different processes," J. Nanomater. Vol.2018, Article ID 2012140, 2018.

[3] Q. Wang, D.G. Yu, S.Y. Zhou, C. Li, and M. Zhao, "Fabrication of amorphous electrospun medicated-nanocomposites using a Teflonbased concentric spinneret," e-Polymer, vol.18, pp.3-11, 2018.

[4] Y.H. Wu, D.G. Yu, J.J. Li, Q. Wang, H.P. Li, and X.Y. Li, "Medicated multiple-component polymeric nanocomposites fabricated using electrospraying," Polym. Polym. Compos. vol.25, pp.57-62, 2017.

[5] Q. Wang, D.G. Yu, L.L. Zhang, X.K. Liu, Y.C. Deng, and M. Zhao, "Electrospun hypromellose-based hydrophilic composites for rapid dissolution of poorly water-soluble drug," Carbohydr. Polym. vol.174, pp.617-625, Oct, 2017.

[6] Y.H. Wu, D.G. Yu, H.P. Li, X.Y. Wu, and X.Y. Li, "Medicated structural PVP/PEG composites fabricated using coaxial electrospinning," e-Polymers, vol.17, pp.39-44, January 2017.

[7] J. Li, Chen Yang, Hai-Peng Li, Qing Wang, and Deng-Guang Yu, "Oral controlled release in accordance with drug adsorption biological rhythm provided by an electrospun structural amorphous solid dispersion," J. Control. Release, vol.259, pp.e61-e62, 2017.

[8] D.G. Yu, H.P. Li, C. Yang, J.J. Li, Q. Wang, and G.R. Williams, "Double-pulsatile release core-shell fibers fabricated using modified tri-axial electrospinning," J. Control. Release, vol.259, pp.e24-e25, 2017.

[9] C. Yang, D.G. Yu, D. Pan, X.K. Liu, X. Wang, S.W.A. Bligh, et al, "Electrospun $\mathrm{pH}$-sensitive core-shell polymer nanocomposites fabricated using a tri-axial processes," Acta Biomater., vol.35, pp.77-86, April 2016

[10] X. Liu, W. Shao, M. Luo, J. Bian, D.G. Yu, "Electrospun blank nanocoating for improved sustained release profiles from medicated gliadin nanofibers," Nanomaterials, vol.8, Article ID 184 (11 pages), 2018.

[11] Y.Y. Yang, M. Zhang, Z.P. Liu, K. Wang, and D.G. Yu, "Meletin sustained-release gliadin nanoparticles prepared via solvent surface modification on blending electrospraying," App. Surf. Sci., vol.434, pp.1040-1047, 2018.

[12] Y.H. WU, H.P. Li, X.X. Shi, J. Wan, Y.F. Liu, and D.G. Yu, "Effective utilization of the electrostatic repulsion for improved alignment of electrospun nanofibers," J. Nanomater., vol.2016, Article ID2067383, 2016.

[13] Z.P. Liu, Y.Y. Zhang, D.G. Yu, D. Wu, and H.L. Li, "Fabrication of sustained-release zein nanoparticles via modified coaxial electrospraying,” Chem. Eng. J., vol.334, pp.807-816, 2018.

[14] Wang, X.K. Liu, X.H. Chen, D.G. Yu, Y.Y. Yang, and P. Liu, "Electrospun hydrophilic Janus nanocomposites for the rapid onset of therapeutic action of helicid," ACS Appl. Mater. Interfaces, vol.10, pp.2859-2867, 2018.

[15] H.F. Wen, C. Yang, D.G. Yu, X.Y. Li, and D.F. Zhang, "Electrospun zein nanoribbons for treatment of lead-contained wastewater," Chem. Eng. J., vol.290, pp.263-272, April 2016.

[16] Y.Y. Yang, Z.P. Liu, D.G. Yu, K. Wang, P. Liu, X. Chen, "Colonspecific pulsatile drug release provided by electrospun shellac nanocoating on hydrophilic amorphous composites," Int. J. Nanomed., vol.2018, DOI: 10.2147/IJN.S154849, 2018.

[17] G.Z. Yang, H.P. Li, J.H. Yang, J. Wan, and D.G. Yu, "Influence of working temperature on the formation of electrospun polymer nanofibers," Nanoscale Res. Lett., vol.12, Article 15, January 2017.

[18] M. Jin, D.G. Yu, C.F.G.C. Geraldes, G.R. Williams, and S.W.A. Bligh, "Theranostic fibers for simultaneous imaging and drug delivery," Mol. Pharm., vol.13, pp.2457-2465, July 2016.

[19] D.G. Yu, C. Yang, M. Jin, G.R. Williams, H. Zou, X. Wang, et al, "Medicated Janus fibers fabricated using a Teflon-coated side-by-side spinneret," Colloid. Surface B, vol.138, pp.110-116, Feburary 2016.

[20] M. Jin, D.G. Yu, X. Wang, C.F.G.C. Geraldes, G.R. Williams, and S.W.A. Bligh, "Electrospun contrast agent-loaded fibers for colontargeted MRI," Adv. Healthcare Mater., vol.5, pp.977-985, April 2016.

[21] D.G. Yu, J.J. Li, M. Zhang, and G.R. Williams, "High-quality Janus nanofibers prepared using three-fluid electrospinning," Chem. Commun., vol.53, pp.4542-4545, April 2017.

[22] G.Z. Yang, J.J. Li, D.G. Yu, M.F. He, J.H. Yang, and G.R. Williams, "Nanosized sustained-release drug depots fabricated using modified triaxial electrospinning," Acta Biomater., vol.53, pp.233-241, April 2017.

[23] Y.H. WU, C. Yang, X.Y. Li, J.Y. Zhu, and D.G. Yu, "Medicated nanofibers fabricated using $\mathrm{NaCl}$ solutions as shell fluids in a modified coaxial electrospinning," J. Nanomater., vol.2016, Article ID 8970213, 2016.

[24] K. Wang, H.F. Wen, D.G. Yu, Y. Yang, and D.F. Zhang, "Electrosprayed hydrophilic nanocomposites coated with shellac for colon-specific delayed drug delivery," Mater. Design, vol.143, pp.248255,2018

[25] Z.P. Liu, L.L. Zhang, Y.Y. Yang, D. Wu,G. Jiang, and D.G. Yu, "Preparing composite nanoparticles for immediate drug release by modifying electrohydrodynamic interfaces during electrospraying," Powd. Technol., vol.327, pp.179-187, 2018.

[26] B. Sanchez-Vazquez, A.J. Amaral, D.G. Yu, G. Pasparakis, and G.R. Williams, "Electrosprayed Janus particles for combined photochemotherapy," AAPS PharmSciTech, vol.8, pp.1460-1468, 2017.

[27] Q. Wang, H.P. Li, C. Yang, J.J. Li, and D.G. Yu, "Beads-on-a-string amorphous solid dispersion fabricated using a modified coaxial electrospinning," J. Control. Release, vol.259, pp.e111-e112, 2017.

[28] X.Y. Li, Z.B. Zheng, D.G. Yu, X.K. Liu, Y.L. Qu, and H.L. Li, "Electrosprayed sperical ethylcellulose nanoparticles for an improved sustained-release profile of anticancer drug," Cellulose, vol.24, pp.5551-5564, 2017.

[29] Y. Xu, J.J. Li, D.G. Yu, G.R. Williams, J.H. Yang, and X. Wang, "Influence of the drug distribution in electrospun gliadin fibers on drugrelease behavior,” Eur. J. Pharm. Sci., vol.106, pp.422-430, August 2017. 